\title{
Defect-enhanced electron field emission from chemical vapor deposited diamond
}

\author{
W. Zhu, ${ }^{\text {a) }}$ G. P. Kochanski, S. Jin, and L. Seibles \\ AT\&T Bell Laboratories, $600^{-}$Mountain Avenue, Murray Hill, New Jersey 07974
}

(Received 15 December 1994; accepted for publication 18 April 1995)

\begin{abstract}
Diamond samples with varying defect densities have been synthesized by chemical vapor deposition, and their field emission characteristics have been investigated. Vacuum electron field emission measurements indicate that the threshold electric field required to generate sufficient emission current densities for flat panel display applications $\left(>10 \mathrm{~mA} / \mathrm{cm}^{2}\right)$ can be significantly reduced when the diamond is grown so as to contain a substantial number of structural defects. The defective diamond has a Raman spectrum with a broadened peak at $1332 \mathrm{~cm}^{-1}$ with a full width at half maximum (FWHM) of $7-11 \mathrm{~cm}^{-1}$. We establish a strong correlation between the field required for emission and the FWHM of the dianond peak. The threshold fields are typically less than 50 $\mathrm{V} / \mu \mathrm{m}$ and can reach as low as $30 \mathrm{~V} / \mu \mathrm{m}$ for diamond with a FWHM greater than $8.5 \mathrm{~cm}^{-1}$. It is believed that the defects create additional energy bands within the band gap of diamond and thus contribute electrons for emission at low electric fields. (c) 1995 American Institute of Physics.
\end{abstract}

\section{INTRODUCTION}

Diamond has attracted much attention as a cold cathode for field emission displays due to its negative electron affinity ${ }^{1}$ and robust mechanical and chemical properties. $\mathrm{Ob}$ servations of electron emission from chemical vapor deposited (CVD) diamond under relatively low electric fields $(3-40 \mathrm{~V} / \mu \mathrm{m})$ have been reported. ${ }^{24}$ Fabrication of diamond field emitter arrays has also been attempted, ${ }^{5}$ and a diodestructured prototype field emission display based on a diamond-like carbon cathode has been demonstrated. ${ }^{6}$ However, it is not entirely clcar how the elcetron emission from these seemingly undoped or $p$-type-doped CVD diamonds can occur at such low fields.

Usually, for a semiconductor field emitter, the emitted electrons can originate from either the conduction band, the valence band, and/or surface states. Diamond has a wide band gap with $E_{g}=5.5 \mathrm{eV}$. In order to induce stable electron field emission from diamond, either the bulk or the surface must first be made conductive. Additionally, to optimally take advantage of diamond's negative electron affinity to realize low-voltage electron emission, the Fermi level must be as high in the gap as possible. Unfortunately, efforts so far in search of effective and reliable $n$-type dopants have not been successful. ${ }^{7,8}$ Common $n$-type dopants in silicon such as nitrogen, phosphorus, and arsenic are found to either occupy too deep a level in diamond to be activated or have very low solubilities in diamond. Although $p$-type semiconducting diamond is readily available by doping with boron, it is not helpful for achieving low-field electron emission because the energy levels filled by electrons are approximately $5 \mathrm{eV}$ below the vacuum level. Surface states do exist in the band gap on reconstructed diamond surfaces, ${ }^{9,10}$ but it is not known how the electrons are transported to these surface states to sustain the emission from the undoped or $p$-type-doped diamonds.

a)Electronic mail: zhu@clockwise.att.com
This study identifies structural properties which govern the electron field emission process from undoped and $p$-typedoped diamonds. Low-voltage diamond field emitters with such properties have been synthesized by controlling CVD process parameters. A strong correlation was established between the field required for emission and the width of the diamond Raman peak. It is believed that the defects create additional energy bands within the band gap of diamond and thus contribute electrons for emission at low electric fields.

\section{EXPERIMENTS AND DATA ANALYSIS}

The diamond samples were prepared by microwave plasma enhanced CVD using a mixture of methane and hydrogen gases. The methane concentration and growth time was systematically manipulated to produce a variety of diamond samples with varying defect densities as listed in Table I. For the undoped samples, the methane was turned off first in the system shutdown procedure, to allow the hydrogen plasma to etch away any graphite that might exist on the surface. $p$-type semiconducting diamond was prepared by bubbling hydrogen through a solution of boron oxide $\left(\mathrm{B}_{2} \mathrm{O}_{3}\right)$ in ethyl alcohol during the growth process. The dopant levels and distribution were measured by secondary ion mass spectroscopy (SIMS). The introduction of both structural defects and boron dopants increases the conductivity and alters the work function of diamond, thus directly affecting the field emission properties of diamond.

Raman spectroscopy was used to evaluate the structural quality of the diamond samples. As shown in Fig. 1, the FWHM of the diamond peak at $1332 \mathrm{~cm}^{-1}$ is a measure of the perfection of the diamond structure (e.g., point defects and stacking faults), and the intensity ratio of the graphitic peak to the diamond peak $\left(I_{g 1} / I_{d 1}\right)$ indicates the amount of graphitic phases present in diamond. ${ }^{11}$ We correlated both the peak width and the intensity ratio with the emission properties and found no clear trend between the electric field necessary for emission and the intensity ratio $\left(I_{g 1} / I_{d 1}\right)$. This indicates that the graphitic defects alone do not account for 
TABLE I. Field emission properties of CVD diamond.

\begin{tabular}{|c|c|c|c|c|c|}
\hline Sample & $\begin{array}{l}\text { Growth } \\
\text { conditions }\end{array}$ & $\begin{array}{c}\text { FWHM of } \\
\text { diamond } \\
\text { Raman } \\
\text { peak }\left(\mathrm{cm}^{-1}\right)\end{array}$ & $\begin{array}{l}\text { Turn-on field } \\
\text { for a current } \\
\text { density of } \\
0.01 \mathrm{~mA} / \mathrm{cm}^{2} \\
(\mathrm{~V} / \mu \mathrm{m})\end{array}$ & $\begin{array}{l}\text { Threshold field } \\
\text { for a current } \\
\text { density of } \\
10 \mathrm{~mA} / \mathrm{cm}^{2} \\
(\mathrm{~V} / \mu \mathrm{m})\end{array}$ & Comments \\
\hline$\# 1$ & $0.4 \% \mathrm{CH}_{4} / \mathrm{H}_{2}, 7 \mathrm{~h}$ & 3.8 & $\cdots$ & $\cdots$ & no emission \\
\hline$\# 2$ & $0.8 \% \mathrm{CH}_{4} / \mathrm{H}_{2}, 45 \mathrm{~h}$ & 4.2 & $\cdots$ & $\cdots$ & no emission \\
\hline$\# 3$ & $1 \% \mathrm{CH}_{4} / \mathrm{H}_{2}, 12 \mathrm{~h}$ & 7.4 & 72 & 120 & $\ldots$ \\
\hline$\# 4$ & $0.5 \% \mathrm{CH}_{4} / \mathrm{H}_{2}, 7 \mathrm{~h}$ & 7.8 & 51 & 84 & $\cdots$ \\
\hline$\# 5$ & $2 \% \mathrm{CH}_{4} / \mathrm{H}_{2}, 4 \mathrm{~h}$ & 10.9 & 22 & 31 & $\cdots$ \\
\hline$\# 6$ & $1 \% \mathrm{CH}_{4} / \mathrm{H}_{2}, 7 \mathrm{~h}$ & 9.4 & 32 & 46 & $\cdots$ \\
\hline$\# 7$ & $\begin{array}{l}1 \% \mathrm{CH}_{4} / \mathrm{H}_{2}, 7 \mathrm{~h} \text {, and } \\
\text { overcoated at }\end{array}$ & 10.2 & 24 & 40 & $\cdots$ \\
\hline$\# 8$ & $\begin{array}{l}8 \% \mathrm{CH}_{4} / \mathrm{H}_{2} \text { for } 15 \mathrm{~m} \\
0.3 \% \mathrm{CH}_{4} / \mathrm{H}_{2}, 30 \mathrm{~h} \text {, } \\
\text { and B doped }\end{array}$ & 3.7 & 111 & 164 & $\begin{array}{l}\text { boron conc. } \\
10^{20} \mathrm{~cm}^{-3}\end{array}$ \\
\hline$\# 9$ & $\begin{array}{l}5 \% \mathrm{C}_{2} \mathrm{H}_{5} \mathrm{OH} / \mathrm{H}_{2} \\
14 \mathrm{~h} \text {, and } \mathrm{B} \text { doped }\end{array}$ & 8.1 & 58 & 107 & $\begin{array}{l}\text { boron conc. } \\
10^{20} \mathrm{~cm}^{-3}\end{array}$ \\
\hline$\# 10$ & $\begin{array}{l}0.6 \% \mathrm{C}_{2} \mathrm{H}_{5} \mathrm{OH} / \mathrm{H}_{2} \\
45 \mathrm{~h}, \text { and } \mathrm{B} \text { doped }\end{array}$ & 9.1 & 16 & 30 & $\begin{array}{l}\text { boron conc. } \\
10^{19} \mathrm{~cm}^{-3}\end{array}$ \\
\hline$\# 11$ & $\begin{array}{l}2.5 \% \mathrm{C}_{2} \mathrm{H}_{5} \mathrm{OH} / \mathrm{H}_{2} \\
18 \mathrm{~h}, \text { and } \mathrm{B} \text { doped }\end{array}$ & 9.2 & 21 & 37 & $\begin{array}{l}\text { boron conc. } \\
>10^{20} \mathrm{~cm}^{-3}\end{array}$ \\
\hline$\# 12$ & $\begin{array}{l}1.5 \% \mathrm{C}_{2} \mathrm{H}_{5} \mathrm{OH} / \mathrm{H}_{2} \\
60 \mathrm{~h} \text {, and } \mathrm{B} \text { doped }\end{array}$ & 10.5 & 21 & 38 & $\begin{array}{l}\text { boron conc. } \\
>10^{20} \mathrm{~cm}^{-3}\end{array}$ \\
\hline
\end{tabular}

${ }^{a}$ Samples \#1, 6, and 7 have an island-type morphology, while all the other samples are continuous films.

the emission characteristics of diamond. We saw a strong correlation of the emission field with the peak width, indicating that other types of defects are at least as important. Scanning electron microscopy (SEM) was used to measure the crystallite size or emitting tip density; we assume that there was one emitter site per crystallite, on average.

The experimental apparatus used for the field emission measurements is shown in Fig. 2. A voltage cycled from zero up to $2 \mathrm{kV}$ was applied to a tungsten anode $(1 \mathrm{~mm}$ in diameter) to collect electrons emitted from the diamond surface. A precision step controller ( $3.3 \mu \mathrm{m}$ step size) was used to control the movement of the probe toward the sample surface,

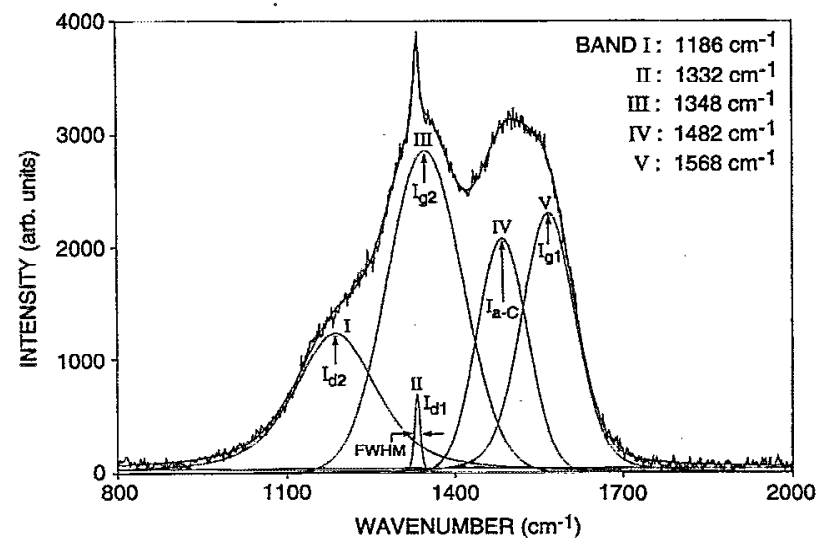

FIG. 1. A representative Raman spectrum with deconvolved components from defective diamond showing the width of the diamond peak at 1332 $\mathrm{cm}^{-1}$ and the intensity ratio of the graphitic peak to the diamond peak $\left(I_{g 1} / I_{d 1}\right)$. Peaks I and III are reported to be associated with the small crystal sizes and/or disordered structures of diamond and graphite, respectively (See Ref. 17). Peak IV is due to the presence of amorphous carbon. and the emission current-voltage $(I-V)$ characteristics was repeatedly measured as a function of the distance between the probe and the diamond surface as shown in Fig. 3. To eliminate the effects of surface contamination on the electron emission from diamond, a hydrogen ion gun $(400 \mathrm{~V}, 0.2$ $\mathrm{mA} / \mathrm{cm}^{2}$ ) was used to gently sputter-clean the sample prior to each measurement. The $I-V$ data were then analyzed using a Fowler-Nordheim equation. The emission current is taken to be an appropriate integral over geometry and a distribution of material parametcrs:

$$
I=2 \pi \int R \int N(\zeta) I_{t}\left[\zeta, E_{m}(R)\right] d \zeta d R
$$

This is necessary because the emitting surface is nonuniform, with electronic properties, curvature, and applied field varying from tip to tip. In Eq. (1), the outer integral is over the surface of the emitter, accounting for the decreasing electrical field as one moves a distance $R$ away from the point directly underneath the spherical anode; $E_{m}(R)$ is the mac-

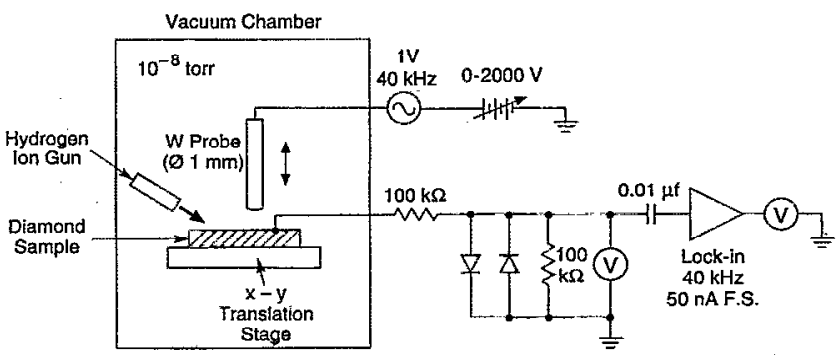

FIG. 2. A schematic diagram of the experimental setup for the field emission measurement. 


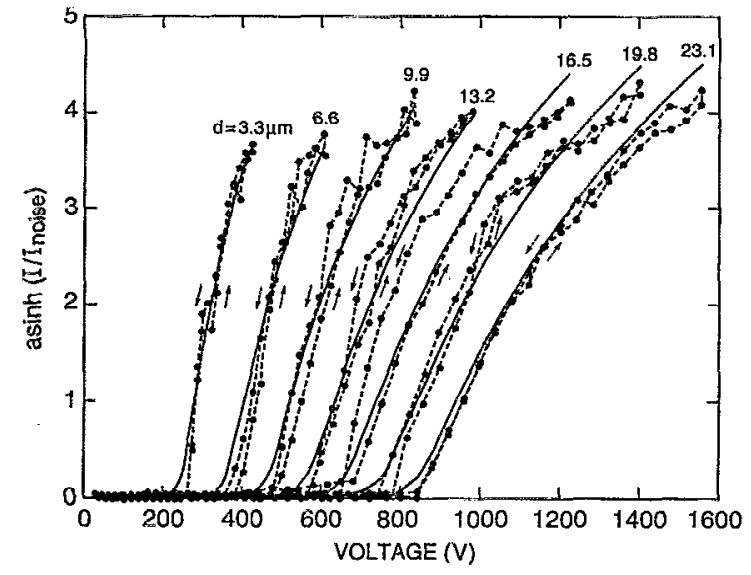

FIG. 3. Representative $I-V$ emission curves from defective CVD diamond obtained at various spacings (d) between the anode and the cathode. The dots and dashed lines are actual data, and the solid lines are fitted curves based on the modified Fowler-Nordheim equation. $I_{\text {noise }}$ is $0.1 \mathrm{nA}$.

roscopic electric field without local field enhancement. ${ }^{12}$ The inner integral is over the probability distribution of tip parameters (e.g., some combination of sharpness and work function); we take $\zeta$ to be the most important combination of sharpness and work function. $N(\zeta)$ is the density of tips per unit area that have parameter $\zeta$, and $I_{t}$ is the current emitted by each such tip. The shape of $N(\zeta)$ is generally unknown, though it is clearly broad (when we place a phosphor screen over the emitter, we find that the emission is dominated by small areas; only $1 \%-10 \%$ of the emitter surface contributes nearly all the current). We have taken $N(\zeta)=N_{0} \exp (-|\zeta|) / 2$, where $N_{0}$ is the tip density obtained from the SEM measurement; a Gaussian distribution function gives similar results.

Because of the nonuniformity, it is likely that the best emission region (which may dominate the emission current) is not directly under the end of the probe. We have introduced a parameter $\alpha$ that specifies how far laterally the hot spot is from the probe; this should vary randomly from location to location. We calculate the effective radius $R_{\text {eff }}$ of an emitting region that would be required to produce $I$ if the current per tip were constant at its central (e.g., $R=0$ ) value; we then mathematically move the tip up by $\alpha^{2} R_{\text {eff }}^{2} f\left(2 R_{\text {probe }}\right)$ (the amount the spherical probe has curved away from the surface at a radius $\alpha R_{\text {eff }}$ ). We find that fitted values of $\alpha$ are typically less than one, as would be expected.

The current per tip, $I_{t}=2 \pi r^{2} \int_{0}^{\pi / 2} \sin \phi$ $\cdot J\left[\zeta, E_{m}(R) \cdot f(\phi)\right] d \phi$ is similarly an angular integral of the local current density over the end of each emitting tip. We take $r$ to be an average tip radius (simply to make units consistent- the value of $r$ collapses into $a_{0}$, below); deviations from the average are collapsed into $a_{1}$ (below) or $\zeta$. Likewise, the part of the field enhancement factor that depends on tip radius is collapsed into $J(. .$.$) via other param-$ eters (i.e., $a_{0}$ and $b_{0}$, below). What is left is the angular dependence of the field enhancement, $f(\phi)$, which is taken from a sphere-on-cone model. ${ }^{12}$

Finally, $J(\ldots)$ is parameterized with the FowlerNordheim form: $J=E^{2} \exp \left[\left(a_{0}+a_{1} \zeta\right)-\left(b_{0}+b_{1} \zeta\right) / E\right]{ }^{13}$ with $a_{0}$ the $\log$ of the typical F-N offset, and $b_{0}$ the typical
F-N slope. As typical, these parameters contain a combination of information on the tip radius, work function, geometry, and surface physics. The parameters $a_{1}$ and $b_{1}$ then measure the width of the probability distribution of emitters and are proportional to the nonuniformity of the emitters. If $a_{1} \approx 1$ or $b_{1} \approx 1$, then one begins to get strong nonuniformity, with tip-to-tip current variations on the order of $100 \%$.

Thereforc, in addition to the two parameters $a_{0}$ and $b_{0}$ which are used to describe the classic Fowler-Nordheim equation, three new parameters $\left(a_{1}, b_{1}, \alpha\right)$ were found necessary to achieve a good fit of the data. The fitting of data to calculations was carried out by means of a weighted leastsquare fit between $\operatorname{asinh}\left(I_{\text {data }} / I_{\text {noise }}\right)$ and $\operatorname{asinh}\left(I_{\text {calculated }} / I_{\text {noise }}\right)$, where $I_{\text {noise }}$ was the observed measurement noise near zero current $\left(10^{-10} \mathrm{~A}\right)$. The hyperbolic arc-sine function was used as a substitute for $\log$ function that has good behavior at zero current. The appropriately fitted parameters are then used to extrapolate the $I-V$ characteristics of emitters to a standard display pixel. Both the threshold electric field required to obtain a current of $1 \mu \mathrm{A}$ over an area of $100 \mu \mathrm{m}^{2}$ (that is, a current density of $10 \mathrm{~mA} / \mathrm{cm}^{2}$ for sufficient phosphor activation) and the turn-on field (for a current density of 0.01 $\mathrm{mA} / \mathrm{cm}^{2}$ ) were subsequently calculated.

\section{RESULTS AND DISCUSSION}

As indicated in Table I, high quality diamond (e.g., samples \#1 and 2), that has low defect densities and a narrow Raman peak (FWHM $<5 \mathrm{~cm}^{-1}$ ), generally does not emit electrons. When the applied electric field increases, electrical breakdown or arcing eventually occurred on the surface due to the highly insulating nature of the high quality diamond. However, the defective diamond (e.g., samples \#3-7) with the Raman peak width broadened to beyond approximately 7 $\mathrm{cm}^{-1}$ exhibited characteristic Fowler-Nordheim emission behavior. Smooth and consistent $I-V$ curves were measured from these samples in a history independent, reproducible manner as shown in Fig. 3. In the figure, the voltage was raised from zero to the maximum $(+2 \mathrm{kV})$ and then decreased to zero. As the probe moved one step closer $(3.3 \mu \mathrm{m})$ to the sample surface, the above voltage cycle was repeated. Curve fitting analysis gives threshold ficld values in the range of $30-120 \mathrm{~V}$ per micron distance between the anodecathode gap and turn-on field in the range of 22-72 V per micron for these defective diamond samples. This compares favorably with the high quality, $p$-type-doped diamond which requires a turn-on field of more than $110 \mathrm{~V} / \mu \mathrm{m}$ and a threshold field of greater than $160 \mathrm{~V} / \mu \mathrm{m}$. A strong correlation between the threshold emission field and the FWHM of the diamond Raman peak is evident as shown in Fig. 4. In the figure, data from ion-implanted diamond samples are also included. $^{14}$

It can be seen that for the field emission to occur, undoped diamond must exhibit a Raman peak width in a narrow range of about $7-11 \mathrm{~cm}^{-1}$. Undoped diamond with the Raman peak width less than $7 \mathrm{~cm}^{-1}$ are generally too insulating to sustain a stable emission. On the other hand, when the peak width exceeds $11 \mathrm{~cm}^{-1}$, the diamond Raman signature becomes so broad and weak compared to peaks originating from graphitic and amorphous carbon components 


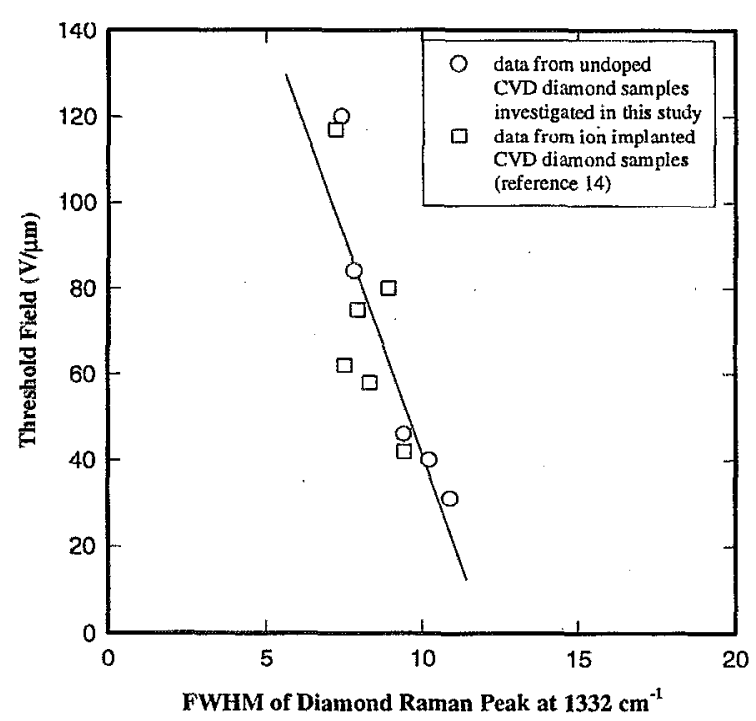

FIG. 4. A diagram showing the correlation between the diamond Raman peak width with the threshold field required to yield an emission current density of $10 \mathrm{~mA} / \mathrm{cm}^{2}$ for the undoped diamond.

that the deposits are usually considered as diamond-like or amorphous carbon films rather than genuine diamond. These films are reported to be capable of emitting electrons at relatively low fields, ${ }^{6}$ but their emission stability and reliability have not be established, and they are not the subject of the present study. For the undoped diamond, the required threshold field for emission falls rapidly as the Raman peak width increases, from about $120 \mathrm{~V} / \mu \mathrm{m}$ at the peak width of 7.4 $\mathrm{cm}^{-1}$ to about $30 \mathrm{~V} / \mu \mathrm{m}$ at $10.9 \mathrm{~cm}^{-1}$.

Such a strong correlation between the emission field and the width of the diamond Raman peak is also observed from the boron-doped, $p$-type semiconducting diamond as shown in Fig. 5. Samples with larger peak width or higher defect

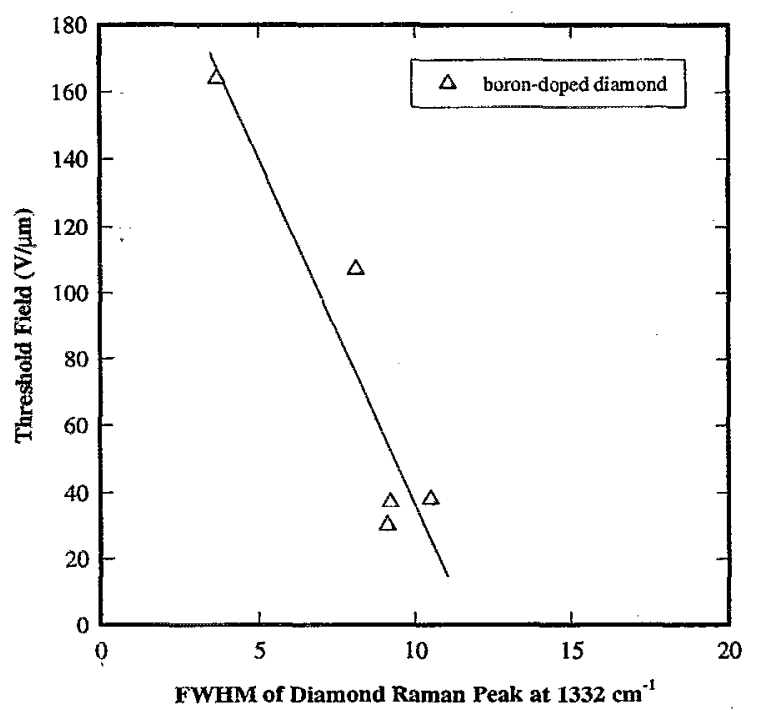

FIG. 5. A diagram showing the correlation between the diamond Raman peak width with the threshold field required to yield an emission current density of $10 \mathrm{~mA} / \mathrm{cm}^{2}$ for the boron-doped, $p$-type diamond.

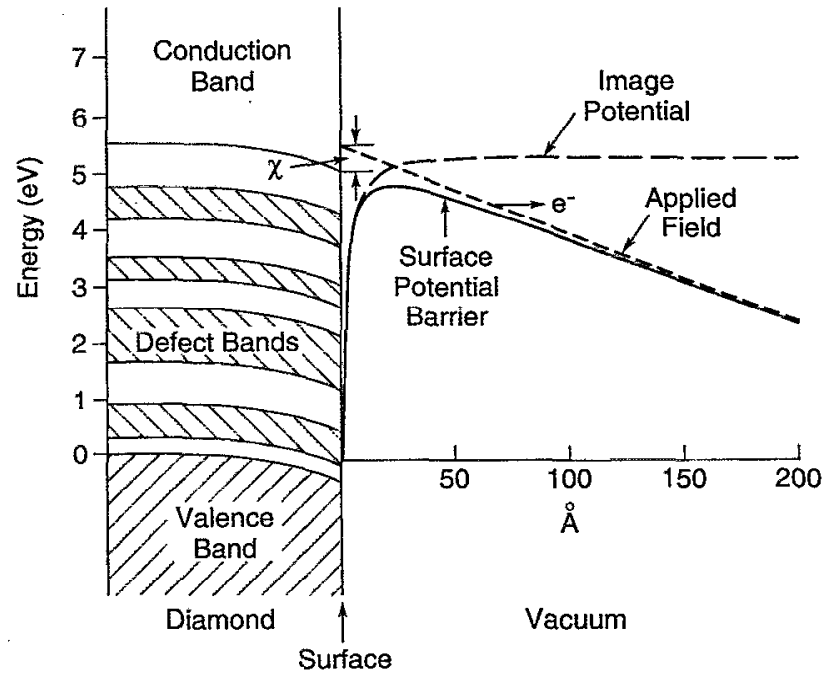

FIG. 6. A schematic energy band diagram showing the presence of defectinduced energy bands wilhin the band gap of diamond. A positive electron affinity $(\chi)$ of $0.5 \mathrm{eV}$ is assumed. The applied field is $150 \mathrm{~V} / \mu \mathrm{m}$ which causes the band bending, and no surface states are shown. The position and width of the defect-induced bands are arbitrary for illustration purpose only.

densities require lower fields for emission. The samples with the peak width in the range of $9.1-10.5 \mathrm{~cm}^{-1}$ (samples \#1012) require a threshold field in the range of $30-40 \mathrm{~V} / \mu \mathrm{m}$. This is almost five times lower than that required for a high quality $p$-type sample (sample \#8) which has a peak width of $3.7 \mathrm{~cm}^{-1}$. It is interesting to note that the $p$-type semiconductivity allows stable emission to be obtained from diamond exhibiting a Raman peak width as low as $3.7 \mathrm{~cm}^{-1}$. If undoped, such high quality diamond would show arcing or breakdown behavior similar to samples \#1 and 2 .

There have been a number of reasons proposed that would relate defect density to field emission characteristics. For instance, emission from graphitic inclusions or enhanced conductivity along grain boundaries have been suggested. ${ }^{3,4}$ However, both our sample growth technique and Raman measurements argue against any contribution from graphite. We believe it is more likely that the enhanced field emission originates from the defect-induced energy band(s) which are formed within the band gap of bulk diamond. ${ }^{15,16}$ As schematically shown in Fig. 6, a series of defect-induced bands are assumed to form throughout the band gap because of the presence of a wide variety of structural defects. If these bands are wide enough or closely spaced, the electron hopping within the band(s) or excitation from the valence band could easily provide a steady flow of electrons to the surface (or surface states which are not identified in the diagram) to sustain stable emission of electrons into vacuum. The formation of these defect bands raises the Fermi level into the upper part of the band gap, and thus reduces the energy barrier that the electrons must tunnel through. However, the exact positions of these defect-induced energy bands can not be determined from the field emission measurements because of uncertainties in the local field enhancement and the emission area, both of which are impossible to quantify at present. Both theoretical calculations and experimental studies such as photoemission are needed to determine how the 
defects couple together to form conducting bands and to identify the exact energy levels from which the electrons originate.

\section{CONCLUSIONS}

In summary, vacuum field emission measurements on CVD diamond samples with varying defect densities indicate that the electric field required for electron emission can be significantly reduced when the CVD diamond is properly grown so as to contain a substantial number of structural defects. A strong correlation is established between the required threshold field to generate sufficient emission current densities for display applications $\left(>10 \mathrm{~mA} / \mathrm{cm}^{2}\right)$ and the $\mathrm{Ra}-$ man peak width which is a measure of the defect densities in diamond. It is likely that the broad types of defects present in the diamond structure create additional energy bands within the band gap of diamond and thus contribute to the electron emission at low electric fields.

${ }^{1}$ F. J. Himpsel, J. A. Knapp, J. A. van Vechten, and D. E. Eastman, Phys. Rev. B 20, 624 (1979).

${ }^{2}$ M. W. Geis, N. N. Efremow, J. D. Woodhouse, M. D. McAleese, M. Marchywka, D. G. Socker, and J. F. Hochedez, IEEE Electron Device Lett. 12, 456 (1991).
${ }^{3}$ C. Wang, A. Garcia, D. C. Ingram, M. Lake, and M. E. Kordesch, Electronics Lett. 27, 1459 (1991).

${ }^{4}$ N. S. Xu, Y. Tzeng, and R. V. Latham, J. Phys. D 26, 1776 (1993).

${ }^{5}$ K. Okano, K. Hoshina, M. Iida, S. Koizumi, and T. Inuzuka, Appl. Phys. Lett. 64, 2742 (1994).

${ }^{6} \mathrm{H}$. K. Schmidt, M. H. Clark, I. Yee, and N. Kumar, Abstract for the SID Manuf. Conference, 1994, p. 21.

${ }^{7}$ G. S. Gildenblat, S. A. Grot, and A. R. Badzian, Proc. IEEE 79, 647 (1991).

${ }^{8} \mathrm{~K}$. K. Das, in Diamond Films and Coatings, edited by R. F. Davis (Noyes, Park Ridge, NJ, 1993), p. 381.

${ }^{9}$ B. B. Pate, M. Oshima, J. A. Silberman, G. Rossi, I. Lindau, and W. E. Spicer, J. Vac. Sci. Technol. A 2, 957 (1984).

${ }^{10}$ B. B. Pate, Surf. Sci. 165, 83 (1986).

${ }^{11} W$. A. Yarbrough and R. Messier, Sci. 247, 688 (1990).

${ }^{12}$ J. He, P. H. Cutler, N. M. Miskovsky, T. E. Feuchtwang, I. E. Sullivan, and M. Chung, Surf. Sci. 246, 348 (1991). Note that equation 63 in this reference makes the approximation that the tip radius is much smaller than the tip-to-anode spacing. This must be generalized if diamond particle sizes are similar to the spacing.

${ }^{13}$ R. H. Fowler and L. W. Nordheim, Proc. R. Soc. London A 119, 173 (1928).

${ }^{14}$ W. Zhu, G. P. Kochanski, S. Jin, L. Seible, D. Jacobson, M. McCormack, and $A$. White (unpublished).

${ }^{15}$ G. B. Bachelet, G. A. Baraff, and M. Schluter, Phys. Rev. B 24, 4736 (1981).

${ }^{16}$ W. V. M. Machado, J. Kintop, M. L. De Siqueira, and L. G. Ferreira, Phys. Rev. B 47, 13219 (1993).

${ }^{17}$ R. J. Nemanich, J. T. Glass, G. Lucovsky, and R. E. Shroder, J. Vac. Sci. Technol. A 6, 1783 (1988). 